\title{
Avaliação econômica em saúde: triagem neonatal da galactosemia
}

\author{
Newborn screening for galactosemia: \\ a health economics evaluation
}

\footnotetext{
1 Faculdade de Medicina de Ribeirão Preto, Universidade de São Paulo, Ribeirão Preto, Brasil.

2 Faculdade de Economia, Administração e

Contabilidade, Universidade de São Paulo, São Paulo, Brasil.

${ }^{3}$ Faculdade de Economia, Administração e

Contabilidade de Ribeirão

Preto, Universidade de São

Paulo, Ribeirão Preto, Brasil.

${ }_{4}^{4}$ Escola de Enfermagem de

Ribeirão Preto, Universidade

de São Paulo, Ribeirão Preto,

Brasil.

Correspondência

J. S. Camelo Junior

Departamento de Puericultura

e Pediatria, Faculdade de

Medicina de Ribeirão Preto,

Universidade de São Paulo.

Av. Bandeirantes 3900 ,

70 andar, Ribeirão Preto

SP 14049-900, Brasil.

jscamelo@fmrp.usp.br
}

\begin{abstract}
This study assesses the efficiency of the galactosemia add-on test in neonatal screening performed on regular Guthrie card blood spots. Based on estimated average incidence of galactosemia (1:19,984 newborns) in São Paulo State, Brazil, the study develops a cost-benefit analysis model, using a B/C ratio and a $9.25 \%$ annual interest rate in order to decapitalize the results. Sensitivity analysis is also performed, varying (as a function of the interest or discount rate) from 0 and $20 \%$ and according to the 95\% confidence interval (1:7,494-1:59,953 newborns). The results show that the savings obtained by improved health of galactosemic patients detected early by add-on neonatal screening is superior to the costs (B/C=1.33), characterizing galactosemia add-on testing in neonatal screening as an efficient policy. The lower the prevailing interest rate in the economy, the more efficient the neonatal screening policy.
\end{abstract}

Health Economics; Cost Efficiency Analysis; Neonatal Screening; Galactosemias
José Simon Camelo Junior 1

Maria Inez Machado Fernandes 1

Salim Moysés Jorge 1

Lea Maria Zanini Maciel 1

Jair Lício Ferreira Santos 1

Alceu Salles Camargo Jr. 2

Cláudia Souza Passador 3

Sílvia Helena Henriques Camelo 4

\section{Introdução}

A saúde no Brasil apresentou mudanças expressivas que provocaram um crescimento acelerado dos gastos em saúde e uma das principais causas foi o desenvolvimento tecnológico de novas estratégias diagnósticas e terapêuticas - que na grande maioria das vezes são mais eficazes, mas ao mesmo tempo mais dispendiosas $1,2,3$.

Torna-se crítico, portanto, para qualquer serviço de saúde, estabelecer políticas bem definidas de adoção, manutenção e incorporação de novas tecnologias no sistema para minimizar os aspectos negativos e, assim, contribuir para uma maior qualidade dos serviços de saúde 4, maximizando-se os ganhos em saúde com o bom uso dos recursos disponíveis. A tomada de decisão deve apoiar-se em avaliações criteriosas que levem em consideração aspectos clínicos e econômicos. É nesse campo que se desenvolve a avaliação econômica em saúde, parte das avaliações de tecnologias em saúde ${ }^{2}$. Países como a Inglaterra, País de Gales e Canadá criaram instituições que se dedicam a tais políticas. No Brasil, o Departamento de Ciência e Tecnologia do Ministério da Saúde tem estimulado o desenvolvimento da avaliação econômica e sua participação na tomada de decisões relacionadas à incorporação de novas tecnologias no Sistema Único de Saúde (SUS) 5.

A avaliação econômica em saúde visa responder basicamente duas grandes questões 2,6: 
"A estratégia de saúde em questão é vantajosa quando comparada com alternativas que poderiam ser implementadas com estes recursos?" 3 e "Estamos cientes e de acordo que os recursos sejam usados desta maneira e não de outra?" 2.

Os principais métodos de avaliação econômica utilizados na área da saúde são: a análise custo-benefício, análise de minimização de custos, análise custo-efetividade e análise custoutilidade 4 . Nosso foco central é a análise custobenefício, que se destaca nas avaliações econômicas, por ser considerada mais abrangente e que contempla todos os aspectos da eficiência alocativa de determinado programa. Por ser um método no qual os custos e benefícios são relatados usando a métrica comum das unidades monetárias, resultados de diferentes estudos são comparáveis e permitem avaliar o quanto a sociedade está disposta a pagar pelos efeitos de programas ou políticas públicas 1 .

Nesse contexto de desigualdades sociais e escassez de recursos para o financiamento da saúde, a avaliação de custo-benefício de ações e serviços é essencial para a elaboração de estratégias e programas que respondam às reais necessidades da população. Embora se possa avaliar eficácia (influência de inovações tecnológicas), efetividade (grau de aproximação aos aprimoramentos possíveis) e eficiência (economia de custos sem prejuízo de metas) ${ }^{7}$, a utilização de avaliações em saúde deve justificar estratégias e programas e auxiliar na racionalização dos gastos públicos e o conceito de eficiência é o nosso foco 7,8 .

Pela utilização dos índices de morbidade e mortalidade infantil como importantes indicadores de saúde do país 9 , observa-se que as causas infecciosas vêm decaindo como responsáveis pela taxa total de óbitos na infância 10,11. À medida que problemas infectocontagiosos estão sendo resolvidos e que se aumenta a capacidade de manter vivos maior número de recém-nascidos, os problemas de ordem congênita e hereditária se tornam pertinentes e de relevância na saúde pública, devendo ser alvo de ações e políticas de saúde específicas.

Atualmente, não há triagem neonatal para galactosemia no Programa Nacional de Triagem Neonatal (PNTN) brasileiro e as crianças que apresentam a doença clássica podem necessitar de tratamento urgente, intensivo, para infecções generalizadas e insuficiência hepática, cirurgias como biópsia hepática, diálise e catarata. Com a política de implementação da triagem neonatal, o Estado estaria economizando os custos de tratamentos de urgência. Tendo sido triadas precocemente, as crianças que apresentam a doença necessitarão de tratamento ambulatorial médico pouco oneroso aos cofres públicos, com mais qualidade de vida e possibilidade real de produtividade social futura, com evolução favorável.

Neste artigo, pretendemos discorrer sobre uma avaliação econômica em saúde que usou a análise custo-benefício da inclusão do diagnóstico da doença metabólica hereditária galactosemia no PNTN, partindo do pressuposto de que a triagem neonatal é uma política pública altamente eficiente de prevenção de retardo mental e morbimortalidade infantil.

\section{A triagem neonatal e a inclusão do exame de galactosemia}

A triagem neonatal é iniciativa de Saúde Pública e Pediatria Preventiva, sendo a maior iniciativa do sistema público de saúde na área de Genética 12. No Brasil, a partir de 1990, começaram a surgir leis relacionadas à triagem neonatal, que culminaram no PNTN em 2001 12,13,14. Foi iniciado em uma primeira fase com a triagem da fenilcetonúria e do hipotireoidismo congênito; em uma segunda fase, acrescentou-se a elas o diagnóstico para hemoglobinopatias. A legislação atual prevê uma terceira fase, com a inclusão da triagem neonatal da fibrose cística, que já ocorre em alguns estados, embora controversa 15.

O PNTN considera que as doenças a serem triadas devem seguir como critérios o fato de não apresentarem manifestações clínicas muito precoces, permitirem a detecção precoce por meio de testes seguros e confiáveis, serem amenizáveis mediante tratamento, serem passíveis de administração em programas com logística definida e terem uma relação custo-benefício economicamente viável e socialmente aceitável 16. Entretanto, tais estudos de custo-benefício jamais foram realizados no Brasil. Os testes vigentes reproduzem modelos e diretrizes internacionais 13 .

Considerando o crescente impacto das doenças genéticas no Brasil e a possibilidade de detecção precoce, acreditamos que cada país deva conhecer a frequência de doenças metabólicas em sua população e realizar os próprios estudos de custo e benefício, para decidir sobre a inclusão ou não dos testes diagnósticos em seu painel obrigatório de screening 13.

É o caso da galactosemia, doença hereditária consequente à deficiência ou ausência de uma das três principais enzimas envolvidas no metabolismo da galactose 17, galactose-1-fosfato-uridiltransferase (GALT), com transmissão autossômica recessiva 16 , sendo conhecidas mais de 230 mutações para o gene GALT 17,18. Manifestações clínicas envolvem insuficiência de crescimento, vômitos, diarreia, icterícia, disfunção hepática, 
anemia hemolítica, podendo progredir com cirrose, insuficiência hepática, cataratas, sinais de hipertensão intracraniana, edema cerebral, letargia, hipotonia e retardo mental. Sepse neonatal fulminante por Escherichia coli é frequente. Muitos pacientes morrem sem diagnóstico ou são diagnosticados tardiamente com danos permanentes. As evoluções agudas incorrem em custos elevados, que poderiam ser evitadas pelo rastreamento e terapêutica adequadas 19,20,21,22,23.

Estimativas da frequência de galactosemia no mundo são variáveis. Nova York (Estados Unidos) e Colúmbia Britânica (Canadá) demonstraram incidência de 1:35.000; 1:60.000 no Reino Unido 13,18,19. Estudos asiáticos demonstram frequência próxima a 1:100.000 21. África do Sul sugere incidência de 1:14.400 recém-nascidos 22 .

Estudo-piloto realizado no Estado de São Paulo com 59.953 recém-nascidos, usando amostragem de $10 \%$ do total de recém-nascidos (603.368 nascidos vivos em 2006) 23 , para a análise da galactosemia no teste do pezinho, identificou uma frequência de 1:19.984, mais elevada, portanto, que a dos caucasianos europeus e norte-americanos. Especulamos que a influência da miscigenação populacional brasileira com afrodescendentes possa ser a possível causa dessa elevada incidência ${ }^{24}$. Os dados foram aleatoriamente obtidos em quatro Centros de Referência de Triagem Neonatal no Estado de São Paulo: Hospital das Clínicas da Faculdade de Medicina de Ribeirão Preto, Universidade de São Paulo (FMRP/ USP) em Ribeirão Preto; Associação dos Pais e Amigos dos Excepcionais (APAE) de Bauru; Centro Integrado de Pesquisas Onco-hematológicas na Infância (CIPOI) da Universidade Estadual de Campinas (UNICAMP) em Campinas; e Hospital Santa Marcelina em São Paulo.

Diante do exposto, sobre a importância potencial da detecção precoce da galactosemia por triagem neonatal e da necessidade de mudanças e novos investimentos que atendam a camadas desfavorecidas e fragilizadas da população, estabelecemos como hipótese que a triagem neonatal para a galactosemia é adequada do ponto de vista de custo-benefício e, fundamentados neste pressuposto, realizamos uma avaliação econômica em saúde.

\section{Métodos}

Esta é uma modelagem para a operacionalização da relação custo-benefício na avaliação da eficiência econômica da política pública de adição da triagem neonatal para a galactosemia 21,25. Consistente com outros métodos de análise econômica de fluxos de caixas, o método de custo-benefício possibilita a separação da quantificação dos custos e dos benefícios obtidos no produto considerado. O cálculo foi feito de forma a apresentar um índice ou coeficiente de benefício/custo (B/C).

Depois da análise e obtenção do fluxo de caixa do projeto de investimento, foi obtida a descapitalização dos benefícios e dos custos, de forma separada, para posteriormente obtermos a relação B/C do projeto pela divisão do valor presente dos benefícios pelo valor presente dos custos:

$$
\frac{B}{C}=\frac{\sum_{j=1}^{J} \frac{B_{j}}{(1+i)^{j}}}{\sum_{k=1}^{K} \frac{C_{k}}{(1+i)^{k}}}
$$

B/C: relação benefício/custo, obtida na data presente ( $t=0$, no fluxo de caixa);

$\mathrm{B}_{\mathrm{j}}$ : valor do benefício, presente no fluxo de caixa do projeto, obtido no instante $\mathbf{j}$;

J: número total de benefícios presentes no fluxo de caixa do projeto;

$\mathrm{C}_{\mathrm{k}}$ : valor do custo, presente no fluxo de caixa do projeto, incorrido no instante k;

K: número total de custos presentes no fluxo de caixa do projeto;

i: taxa de juros utilizada para a descapitalização dos valores de benefícios e custos.

A relação B/C pode assumir para determinado projeto um valor positivo, sendo a decisão do administrador baseada nesse resultado. Se a relação mostrar-se superior à unidade, o projeto é, então, considerado eficiente e o investimento deve ser realizado.

A incidência de galactosemia utilizada para os cálculos da razão $\mathrm{B} / \mathrm{C}$ foi a obtida por em estudo nacional recente 24 , com intervalos de $95 \%$ de confiança (IC95\%) calculados pelo método exato-binomial de Poisson 26.

Para efeito de simplificação da operacionalização do modelo de custo-benefício, foi realizada a comparação entre dois contextos: o atual, sem a inclusão da triagem neonatal para galactosemia e o com a inclusão da referida triagem. Os dados empregados para os cálculos dos benefícios e dos custos foram de naturezas médicohospitalares e econômicos e considerados os seguintes aspectos:

- Custo da triagem por teste, o que permite o cálculo do custo do diagnóstico por paciente detectado por ano. Estão incluídos: (i) custo do kit, fornecido por Intercientifica (São José dos Campos, Brasil); (ii) custo da busca ativa - contato telefônico, transporte do paciente e seus acompanhantes e perda de dia de trabalho dos acompanhantes (perda de produtividade); (iii) custo da confirmação da doença, incluindo o valor da consulta hospitalar, da determinação enzimática 
da GALT e do transporte; e (iv) tratamento e monitoramento ambulatorial.

- Custo do diagnóstico tardio do número estimado de casos detectáveis por ano, baseado na incidência referida 24 . Foram incluídos: (i) custos das internações em enfermarias e unidades de terapia intensiva, sendo o número de dias baseado na média histórica dos casos de galactosemia diagnosticados nas Unidades de Terapia Intensiva Neonatal e Pediátrica, enfermarias de Pediatria e ambulatórios de Gastroenterologia Infantil do HC-FMRP/USP em 20 anos de seguimento; (ii) custos de procedimentos cirúrgicos necessários (biópsia hepática e cirurgias de catarata); (iii) custo do tratamento dietético - fornecimento de latas de fórmulas à base de proteína de soja purificada, sem galactose; e (iv) custo de seguimento ambulatorial em longo prazo, baseado em percentuais de incidência de complicações 19 - para acompanhamento em longo prazo, os pacientes foram divididos em faixas etárias: 0-2 anos, 2-3 anos, 3-10 anos, 1020, anos e 20-65 anos, considerando 65 anos a expectativa de vida de um paciente diagnosticado e tratado.

- Custo do diagnóstico precoce (triagem neonatal) do número estimado de casos detectáveis por ano. Foram incluídos: (i) custo de seguimento ambulatorial em longo prazo, sem complicações - para acompanhamento em longo prazo, os pacientes foram divididos em faixas etárias referidas acima, com a mesma expectativa de vida; e (ii) custo do tratamento dietético - fornecimento de fórmulas à base de proteína de soja purificada, sem galactose.

A diferença entre o custo da triagem e custo do diagnóstico tardio é considerada benefício.

Benefícios com ganho de produtividade dos pacientes triados, em relação aos de diagnóstico tardio, que evoluem com sequelas e não poderão trabalhar no futuro: o ganho de produtividade avalia que o paciente triado ou sem sequelas poderá trabalhar normalmente entre os 20 e os 65 anos de idade e para computar os ganhos, utilizamos o rendimento médio mensal real das pessoas ocupadas no Brasil em março de 200927.

Os custos indexados do atendimento ambulatorial e de internações foram baseados no Relatório Anual do Centro de Custos Especiais do HC-FMRP/USP e envolvem: (i) pessoal, encargos e benefícios; (ii) consumo de materiais, medicamentos etc.; (iii) rateios de contas-correntes de apoio (rouparia, costura, entre outros serviços de apoio); (iv) serviços de terceiros (vigilância, creche, processamento de dados); e (v) outras despesas relacionadas à estrutura hospitalar (água, energia, telefone etc.) e depreciação de bens.
Posteriormente, procedemos à análise de sensibilidade por modelo multivariado, para capturar os efeitos da taxa de juros sobre a relação econômica B/C, em função da incidência estimada e IC95\% pelo método de Poisson 28. O método de análise de sensibilidade é parte fundamental de qualquer avaliação econômica em saúde e é utilizada para se inferir o comportamento de um sistema ou fenômeno sob condições diversas de ocorrência, desde o melhor cenário até o mais desfavorável. Uma vez que os parâmetros utilizados em um modelo variam no mundo real, faz-se necessário avaliar qual impacto dessas variações nos resultados encontrados 29 .

O trabalho foi aprovado pelo Comitê de Ética em Pesquisa do HC-FMRP/USP e os responsáveis pelas crianças participantes assinaram um Termo de Consentimento Livre e Esclarecido, de acordo com a Declaração de Helsinki e Resolução CNS no. 196/96 (processo HCRP no. 7958/2004).

\section{Resultados}

Dentre as 59.953 crianças rastreadas, foram encontrados 158 testes positivos para a galactose total na primeira amostra. Essas crianças foram convocadas para uma segunda triagem. Foram feitas as segundas amostras e apenas 11 mantiveram-se positivos, necessitando da confirmação enzimática da doença. Identificaram-se, então, três crianças com a galactosemia clássica, definindo a incidência da doença em 1:19.984 nascidos vivos.

Os custos incrementais da política de implementação da triagem neonatal da galactosemia são:

- Kit: R\$2.700,00 / 2.000 determinações;

- Recall: contato telefônico de R\$2,00, transporte (criança e um acompanhante) $\mathrm{R} \$ 5,00$ e a perda de produtividade (meio dia de trabalho) $\mathrm{R} \$ 21,65$, com custo total de $\mathrm{R} \$ 48.261,10$.

Considerando-se a incidência $0,01833 \%$, em relação às 59.953 triadas, aproximadamente 110 crianças deverão retornar para o teste de confirmação e à consulta médica para avaliação clínica: desses procedimentos obtém-se o custo de $\mathrm{R} \$ 100,00$ por teste de confirmação, $\mathrm{R} \$ 80,34$ para a consulta médica no HC-FMRP/USP, R $\$ 2,00$ de contato telefônico, $\mathrm{R} \$ 5,00$ para o meio de transporte e $\mathrm{R} \$ 21,65$ para a perda de produtividade (meio dia de trabalho). Sendo, portanto, um custo real de $\mathrm{R} \$ 22.768,35$.

Como há cerca de 600 mil nascidos anualmente no Estado de São Paulo e o custo por determinação de galactose total é de $\mathrm{R} \$ 1,52$ (Kit 
NeoLisa Total Galactose - Intercientifica), temos um valor total de custo de $\mathrm{R} \$ 912.000,00 /$ ano.

Uma nova convocação para a realização da triagem em segunda amostra também gera custos. Foi utilizada aqui a incidência $0,26333 \%$, do total de 59.953 crianças triadas. Do total anual de recém-nascidos em São Paulo, estimamos que aproximadamente 1.580 crianças deverão retornar para um segundo exame. Os custos de contato telefônico e de transporte foram estimados em valores médios. O valor da perda de produtividade foi considerado como a perda de meio dia de trabalho ( $\mathrm{R} \$ 21,65)$, com base no rendimento médio mensal real das pessoas ocupadas no Brasil em março de 2009, R\$ 1.298,70 27.

Assim, ao levarmos em consideração o custo total incorrido anualmente com a política de implementação da triagem neonatal para galactosemia, somando-se os totais de gastos descritos anteriormente é de R\$983.029,45. Há de se fazer a descapitalização desse custo imaginando-se que ele seja igualmente distribuído por doze meses. Para tanto, empregamos a taxa de juros SELIC (Sistema Especial de Liquidação e de Custódia) de 9,25\% (0,74\% ao mês), de junho de 2009 30. O valor do custo total descapitalizado é de R\$937.336,54.

A política de implementação da triagem neonatal de galactosemia introduz benefícios em relação à economia por evitar tratamentos mais frequentes. Se houver a triagem e a doença for detectada, a freqüência de consultas e acompanhamento poderá ser menor para o paciente, apesar de perdurar a vida toda. Logo, do total anual de recém-nascidos em São Paulo, aproximadamente 30 crianças doentes não rastreadas no período neonatal poderiam estar necessitando de tratamentos de urgência.

As crianças não rastreadas com diagnóstico tardio de galactosemia ficam internadas, em mé- dia 4 dias em UTI Neonatal ou Pediátrica e 35 dias em enfermaria de Pediatria, sendo necessárias em média 1,29 cirurgias por criança detectada tardiamente. O custo médio diário em UTI é de $\mathrm{R} \$ 1.499,17$, o custo médio diário de enfermaria comum é R\$515,24 e o custo médio de cirurgias necessárias é de R \$582,95. O valor desses benefícios por evitar os tratamentos de emergência dos casos crônicos, já descapitalizados, é de R $\$$ 709.472,72.

Os benefícios totais por reduzir a frequência do acompanhamento médico do paciente por ter detectado precocemente a doença são de R\$ 35.892,50, já descapitalizados.

O diagnóstico precoce permitido pela triagem de rotina gera melhoria de qualidade de vida do paciente que, potencialmente, poderá trabalhar e tornar-se produtivo para a sociedade. Consideramos que o paciente trabalharia entre 20 e 65 cinco anos de idade e, para computar os ganhos, utilizamos o rendimento médio mensal real das pessoas ocupadas no Brasil de R\$ 1.298,70 em março de 2009 27. Dentre os 30 pacientes que apresentariam a doença anualmente em São Paulo, estima-se que 13 deles apresentariam condições de trabalhar, mesmo com diagnóstico tardio. Portanto, para o cômputo do benefício da política de triagem neonatal, consideramos somente a diferença e, consequentemente, os ganhos de produtividade no trabalho dos outros 17 pacientes. Tais benefícios já descapitalizados apresentariam o valor de R\$449.440,27. Obtivemos, portanto, um valor presente para o total de benefícios de $\mathrm{R} \$ 1.244 .805,49$. A Tabela 1 resume os custos e os benefícios totais.

A relação B/C para o projeto de implementação da triagem neonatal para galactosemia pode, então, ser obtida pela divisão do valor presente total dos benefícios em relação ao valor presente total dos custos. Assim, nosso modelo de

Resumo do total de economias ou benefícios e custos, expressos em reais (R\$) e a razão B/C gerada, em função da frequência média estimada da galactosemia e limites superior e inferior do intervalo de $95 \%$ de confiança, calculado pelo método de Poisson, com taxa de juros de $9,25 \%$ ao ano.

\begin{tabular}{|c|c|c|c|}
\hline & \multicolumn{3}{|c|}{ Incidência } \\
\hline & $1: 7.494$ & $1: 19.984$ & $1: 59.953$ \\
\hline Benefícios com internações e cirurgias & $1.891 .927,25$ & $709.472,72$ & $236.486,96$ \\
\hline Benefícios com tratamento e monitoramento & $95.713,32$ & $35.892,50$ & $11.963,97$ \\
\hline Benefícios com ganho de produtividade & $1.331 .840,73$ & $499.440,27$ & $166.477,31$ \\
\hline Benefícios totais & $3.319 .481,30$ & $1.244 .805,49$ & $414.928,24$ \\
\hline Custos totais & $937.336,54$ & $937.336,54$ & $937.336,54$ \\
\hline Razão B/C & 3,54 & 1,33 & 0,44 \\
\hline
\end{tabular}


avaliação obteve o valor de 1,33 para B/C. Para considerar o impacto da variação da taxa de juros e da incerteza na taxa de incidência da galactosemia sobre o valor da relação $\mathrm{B} / \mathrm{C}$, da política de adição da triagem neonatal para a galactosemia no exame do pezinho, procedemos à análise de sensibilidade, empregando taxas de juros no intervalo entre 0 e $20 \%$ ao ano (recomendado pela Organização Mundial da Saúde (OMS) é entre 3\% e $10 \%$ ao ano) 28,31 .
A incidência da galactosemia encontrada em nossa pesquisa é de 0,000050039 (3 casos em 59.953 recém-nascidos), com intervalos de $95 \%$ de confiança calculados pelo método de Poisson 26 de 1:7.494 a 1:59.953 recém-nascidos. Na análise de sensibilidade, procuramos observar os efeitos da taxa de juros sobre a relação B/C, em função dos valores da média estimada e do IC95\% pela distribuição de Poisson (Tabela 2; Figura 1).

Tabela 2

Efeitos da taxa de juros e da taxa de incidência da galactosemia (incidência média e intervalo de confiança pelo método de Poisson) sobre a relação benefício/custo da inclusão do exame neonatal para galactosemia no Teste do Pezinho.

\begin{tabular}{lccc}
\hline Taxa de juros anual (\%) & $\mathbf{1 : 7 . 4 9 4}$ & $\begin{array}{c}\text { Incidência } \\
\mathbf{1 : 1 9 . 9 8 4}\end{array}$ & $\mathbf{1 : 5 9 . 9 5 3}$ \\
\hline 0,00 & 34,56 & 12,96 & 4,32 \\
3,00 & 12,15 & 4,56 & 1,52 \\
5,00 & 7,20 & 2,70 & 0,90 \\
9,25 & 3,54 & 1,33 & 0,44 \\
10,00 & 3,28 & 1,23 & 0,41 \\
20,00 & 2,20 & 0,83 & 0,28 \\
\hline
\end{tabular}

Figura 1

Efeitos da taxa de juros anual e também da taxa de incidência da doença sobre o benefício/custo da inclusão da triagem neonatal para galactosemia no Teste do Pezinho.

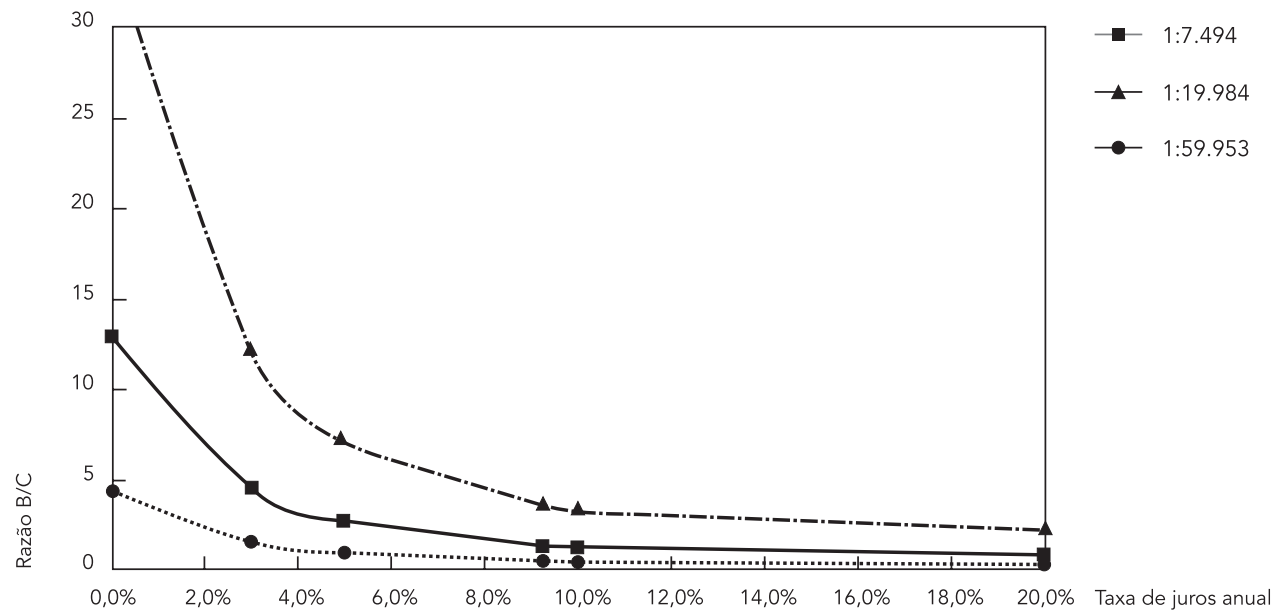

Incidência média estimada = 1:19.984 recém-nascido [intervalo de 95\% de confiança - IC95\%, calculado pelo método de Poisson: 1:7.494 (incidência máxima)-1:59.953 (incidência mínima)]. 


\section{Discussão}

Este trabalho demonstra a eficiência na alocação dos investimentos públicos em um modelo de avaliação econômica em saúde pública, o da implementação da política de inclusão do exame neonatal para a galactosemia no PNTN.

A morbidade e mortalidade infantil fornecem subsídios para os processos de gestão e avaliação de eficiência de políticas e ações de saúde, associados a fatores como saúde materna, qualidade e acesso a serviços de saúde, condições socioeconômicas e práticas de saúde pública ${ }^{9}$. A mortalidade infantil vem concentrando-se nos períodos peri e neonatal, resultando no aumento da proporção de mortes atribuíveis aos defeitos congênitos e doenças metabólicas 11,32. Tal dado também pode ser observado no Brasil, entre 1980 e 2000, quando houve grande redução proporcional dos óbitos por causas infecciosas e respiratórias, passando às más-formações congênitas de quinta para segunda causa de mortalidade infantil 9. Portanto, os problemas de ordem congênita e hereditária se tornam pertinentes e de relevância na saúde pública, devendo ser alvo de ações e políticas de saúde específicas.

Observamos um investimento crescente no setor da saúde que, no entanto, não tem sido suficiente para garantir um atendimento com padrão de qualidade. A avaliação econômica de custo-benefício é uma das técnicas de análise econômico-financeira de fluxo de caixa descontado que pode ser usada como uma medida de eficiência na alocação dos recursos no setor público 33,34. É importante ressaltar que a metodologia do custo-benefício, quando empregada para análise de investimentos no setor público, deve ter em conta todas as benfeitorias que tal projeto estaria introduzindo direta ou indiretamente na sociedade e na economia 29 . Assim, se a relação $\mathrm{B} / \mathrm{C}$ for inferior à unidade, o projeto se mostra ineficiente do ponto de vista de alocação de recursos.

O modelo utilizado 21,25 demonstra vantagem econômica em longo prazo na realização da triagem neonatal para galactosemia, desde que se considerem alguns fatores. $\mathrm{O}$ primeiro fator é a alta incidência populacional. A incidência descrita neste estudo (1:19.985; IC95\%: 1:7.494-1:59.953) é mais elevada que a descrita na Grécia (1:22.182) e Irlanda (1:23.500) 13,35 e apenas inferior à incidência estimada na África do Sul (1:14.400) 22. Isso pode estar ligado à crescente miscigenação da população brasileira. Dados de 2004 da Fundação SEADE, de São Paulo, revelam que a população local apresentava $28,3 \%$ de afrodescendentes, número que vem aumentando (25,5\% em 1991) ${ }^{36}$. Dissertação de
Mestrado recente relata $49 \%$ de afrodescendentes autodeclarados na região de Ribeirão Preto, em 200837.

O segundo fator é a taxa de juros vigente na economia. Considerando a taxa de incidência para a galactosemia utilizada 24 , a relação $\mathrm{B} / \mathrm{C}$ já seria um tanto superior $(B / C=2,70)$ se a taxa fosse de $5 \%$ ao ano, mostrando um forte impacto da taxa de juros que captura os benefícios futuros em valores presentes. Logo, é a taxa de juros vigente na economia que determina as vantagens da triagem incremental em longo prazo, e uma movimentação descendente da taxa na economia torna os benefícios futuros superiores aos custos envolvidos.

Análise de sensibilidade da razão $\mathrm{B} / \mathrm{C}$ demonstra vantagem evidente com taxas de juros inferiores a $10 \%$ ao ano. Valores de economias de países desenvolvidos da Europa e América do Norte, geralmente entre $2 \%$ e $5 \%$ ao ano, recomendados pela OMS 31 , apresentariam B/C altamente vantajosa.

Atualmente, não há triagem neonatal para galactosemia na triagem neonatal obrigatória no Brasil, exceto o Estado de Santa Catarina e no Distrito Federal que determinam essa triagem por legislação local. Com a política de implementação da triagem neonatal, o Estado estaria economizando os custos de tratamentos de emergência, o que foi avaliado, no modelo utilizado neste estudo, como benefícios que advêm de ganhos obtidos com a nova realidade ambulatorial e de saúde e qualidade de vida das crianças.

É interessante, ainda, compararmos a incidência da galactosemia com a fenilcetonúria (PKU). A PKU tem incidência descrita de 1:19.409 nascidos vivos entre 1994 e 2005, no Centro de Referência de Triagem Neonatal do HC-FMRP/ USP 38. Estudo realizado no Paraná, entre 2001 e 2006, demonstra incidência de PKU de 1:20.529 nascidos vivos ${ }^{39}$. Portanto, a incidência da PKU e galactosemia são muito semelhantes em São Paulo e no Paraná, sendo que o tratamento da galactosemia é muito mais barato e tão efetivo quanto o da PKU. Essa análise puramente matemática não considerou, até o momento, o grande impacto social de menor número de casos de crianças e adultos incapacitados pela doença, dependentes não apenas do serviço público, mas de organizações não governamentais que amparam pacientes deficientes físicos e mentais. A redução desse custo social intangível não está incluída 29.

Em favor do diagnóstico precoce, Waisbren et al. 40 compararam 139 crianças com doenças genéticas metabólicas identificadas por triagem neonatal e 124 identificadas por seus sintomas clínicos. Foram encontrados 17 casos de galacto- 
semia no grupo da triagem e 9 no grupo seletivo. Apesar da similaridade na taxa de hospitalizações (66\% versus $77 \%$ ), $47 \%$ das crianças identificadas seletivamente tinham retardo mental, contra $14 \%$ do grupo da triagem, $29 \%$ dos pais do grupo seletivo referiam maiores dificuldades para atender às necessidades de seus filhos versus $7 \%$ do grupo-triagem, havendo mais estresse entre os pais do grupo seletivo contra o grupo triado. Mesmo quando crianças com doenças associadas a internações recorrentes são comparadas, o impacto da triagem neonatal na evolução das crianças e suas famílias é substancial, o que consideramos economia dos custos intangíveis 29 . Esse achado pode ser relevante nas decisões a respeito da expansão da triagem neonatal para doenças genéticas metabólicas adicionais, aumentando-se as oportunidades de melhorias para os pacientes e suas famílias 41 . A identificação precoce e o acompanhamento próximo de doenças como a galactosemia permitem conhecimento mais profundo de fatores moduladores da relação genótipo/fenótipo.

A galactosemia é um bom exemplo de um erro cometido pelas autoridades que acreditam que a triagem para doenças que se apresentam clinicamente no período neonatal não é necessário. A galactosemia pode apresentar-se usualmente na primeira semana de vida com sepse e icterícia, entretanto, muitos pacientes morrem sem diagnóstico ou são diagnosticados tardiamente, quando já ocorreram danos permanentes, situação que ocorre quando o clínico assume erroneamente algum outro processo de base, ou ainda com genótipos mais benignos, de origem africana, que retardam o diagnóstico 19,25,42,43.

A triagem neonatal iniciou-se em 1976 no Brasil com iniciativas isoladas, sem política governamental. A criação do PNTN trouxe uma nova perspectiva para o rastreamento provido pelo sistema público. Foram obtidos dados oficiais sobre as doenças avaliadas, bem como foram criadas condições para manejo dos casos identificados nos Serviços de Referência em Triagem Neonatal que contam com equipes multidisciplinares. Até 2005, o PNTN testou 13 milhões de recém-nascidos, sendo a cobertura da triagem de 80,2\% em 2005 (55\% em 2001) 44.

Nos anos 70, teve início a triagem neonatal na América Latina, que se caracteriza por grande diversidade geográfica, demográfica, étnica, econômica, social e em seus sistemas de saúde. O Brasil representa $42,6 \%$ da área geográfica total e sua população representa $34 \%$ do total. As menores taxas de nascimento estão na Argentina, Chile, Costa Rica, Cuba e Uruguai, mas os quatro primeiros são os pioneiros na organização de programas de triagem neonatal em nível nacional. A cobertura dos programas de rastreamento é de $64 \%$ na Argentina, $100 \%$ no Chile, $98,3 \%$ na Costa Rica e 99,5\% em Cuba e no Uruguai. O país cujo governo realiza o maior investimento em saúde pública é a Argentina (US\$ 358 per capita), seguido por Cuba, Costa Rica, México, Panamá, Uruguai e Chile, com investimentos variando entre US\$ 160 e US\$ 193 per capita. Argentina e a Costa Rica fazem a triagem neonatal para a galactosemia, com frequência estimada de 1:41.549 crianças recém-nascidas 45,46.

Dentre os países europeus, sete faziam a triagem rotineira para a galactosemia em 2004 (Áustria, Alemanha, Hungria, Irlanda, Liechtenstein, Suécia e Suíça), enquanto outros quatro apresentavam programas-piloto ou o programa estabelecido em parte dos laboratórios do país (Turquia, Espanha, Itália e Bélgica) 47. A Holanda iniciou a triagem da galactosemia a partir de 2007; apenas duas doenças metabólicas foram ali estudadas antes da introdução no programa nacional de triagem neonatal, que são a galactosemia e o defeito de $\beta$-oxidação de ácidos graxos de cadeia média, sendo feitas atualmente a triagem de 17 doenças, 12 delas metabólicas 48 .

Nos Estados Unidos, todos os 50 estados realizam rotineiramente a triagem neonatal para a galactosemia no painel mínimo, sendo importante considerar que cada estado tem autonomia para definir o próprio painel 49,50. O Canadá oferece a triagem rotineira para a galactosemia em sete províncias e de maneira seletiva nas demais províncias e territórios 49,51.

Na Ásia e Pacífico, a Nova Zelândia, Austrália, Japão, Filipinas e Taiwan já incluíram a galactosemia nos programas populacionais de triagem neonatal, a despeito de estudos descrevendo a baixa incidência da doença em populações orientais. China e Coréia do Sul oferecem a triagem opcional em alguns estados 21,52.

Corroborando com as iniciativas de diversos países explicitados acima, entendemos que a inclusão do teste da galactosemia no PNTN no Brasil representará um caráter de maior eficiência na alocação de recursos ao gerar economias e saldo positivo aos cofres públicos, além do impacto social positivo não contabilizado na prevenção e detecção precoce dessa doença. 


\section{Resumo}

Este trabalho avalia a eficiência da adição do exame da galactosemia junto ao Teste do Pezinho. Baseado na incidência média estimada de galactosemia, de 1:19.984 recém-nascidos, no Estado de São Paulo, Brasil, este estudo desenvolve um modelo de análise de custo-benefício, utilizando a relação benefício/custo (B/C), a taxa de juros de 9,25\% ao ano para descapitalização dos resultados obtidos. Também se realiza uma análise de sensibilidade, em função da variação da taxa de juros entre 0 e $20 \%$ e do intervalo de $95 \%$ de confiança da incidência da galactosemia (1:7.494 a 1:59.953 recém-nascidos). A economia obtida com a melhora da saúde das crianças doentes identificadas precocemente é superior aos custos $(B / C=1,33)$, caracterizando como eficiente a política de adição do exame neonatal para galactosemia no Teste do Pezinho. Quanto menor a taxa de juros vigente na economia, mais eficiente é a política de triagem neonatal, não considerados os custos sociais intangíveis evitados.

Economia da Saúde; Análise Custo-Eficiência; Triagem Neonatal; Galactosemias

\section{Colaboradores}

J. S. Camelo Junior coordenou o projeto e participou da concepção do projeto, análise e interpretação dos dados, redação do artigo, revisão crítica relevante do conteúdo intelectual e aprovação final da versão a ser publicada. M. I. M. Fernandes, S. M. Jorge, L. M. Z. Maciel, J. L. F. Santos, A. S. Camargo Jr., C. S. Passador e S. H. H. Camelo contribuíram com a análise e interpretação dos dados, redação do artigo, revisão crítica relevante do conteúdo intelectual e aprovação final da versão a ser publicada.

\section{Agradecimentos}

À Karla Panice Pedro (APAE Bauru), Fábio Valdetaro (Hospital Santa Marcelina, São Paulo), Sílvia Brandalise, Carmem Sílvia Gabetta e Daniela Faroro Giovannetti (CIPOI/UNICAMP, Campinas), Roberto Giugliani, Maira Burin e Janice Coelho (HCPA/UFRGS, Porto Alegre). À Fundação de Amparo à Pesquisa do Estado de São Paulo (FAPESP).

\section{Referências}

1. Área de Economia da Saúde e Desenvolvimento, Secretaria Executiva, Ministério da Saúde. Avaliação econômica em saúde: desafios para gestão no Sistema Único de Saúde. Brasília: Ministério da Saúde; 2008.

2. Drummond MF, Sculpher MJ, Torrance GW, O’Brien BJ, Stoddart GL. Methods for the economic evaluation of health care programmes. New York: Oxford University Press; 2005.

3. Krauss-Silva L. Avaliação tecnológica em saúde: questões metodológicas e operacionais. Cad Saúde Pública 2004; 20 Suppl 2:S199-207.

4. Melo JC, Neto MC. Avaliação de Tecnologias da Saúde (ATS) e suas implicações na prática clínica. Einstein (São Paulo) 2008; 6(3 Pt 2):132-4.

5. Departamento de Ciência e Tecnologia, Secretaria de Ciência, Tecnologia e Insumos Estratégicos, Ministério da Saúde. Diretrizes metodológicas: estudos de avaliação econômica de tecnologias em saúde. Brasília: Ministério da Saúde; 2009. (Série A: Normas e Manuais Técnicos).

6. Brouwer WBF, Culyer AJ, van Exel NJA, Rutten FFH. Welfarism vs. extra-welfarism. J Health Econ 2008; 27:325-38.
7. Ribeiro JM, Siqueira SAV, Pinto LFS. Avaliação da atenção à saúde da criança (0-5 anos) no PSF de Teresópolis (RJ) segundo a percepção dos usuários. Ciênc Saúde Coletiva 2010; 15:517-27.

8. Matida AH, Camacho LAB. Pesquisa avaliativa e epidemiologia: movimentos e síntese no processo de avaliação de programas de saúde. Cad Saúde Pública 2004; 20:37-47.

9. Kaye CI; Committee on Genetics, Accurso F, La Franchi S, Lane PA, Hope N, et al. Newborn screening fact sheets. Pediatrics 2006; 118:e934-63.

10. Horovitz DDG, Llerena Jr. JC, Mattos RA. Atenção aos defeitos congênitos no Brasil: panorama atual. Cad Saúde Pública 2005; 21:1055-64.

11. Leite AJM, Almeida NMGS. Mortalidade perinatal: situação atual e perspectivas futuras. In: Procianoy RS, Leone CR, organizadores. ProRN - Programa de Atualização em Neonatologia. Porto Alegre: Artmed Editora; 2008. p. 119-46. (Ciclo 5, Módulo 4).

12. Human Genetics Programme, World Health Organization. Community genetic services in Latin America and regional networks on medical genetics. Report of a WHO consultation. Geneva: World Health Organization; 2004. 
13. Pollitt RJ, Green A, McCabe CJ, Booth A, Cooper NJ, Leonard JV, et al. Neonatal screening for inborn errors of metabolism: cost, yield and outcome. Health Technol Assess 1997; 1:137-40.

14. Ministério da Saúde. Portaria GM/MS no. 22 de 15 de janeiro de 1992. Trata do programa de diagnóstico precoce do hipotireoidismo congênito e fenilcetonúria. Diário Oficial da União 1992; 16 jan.

15. Ministério da Saúde. Portaria GM/MS no. 822 de 6 de junho de 2001. Institui, no âmbito do Sistema Único de Saúde, o Programa Nacional de Triagem Neonatal/PNTN. Diário Oficial da União 2001; 7 jun.

16. Ministério da Saúde. Programa Nacional de Triagem Neonatal. Média e alta complexidade. http:// portal.saude.gov.br/portal/sas/mac/area.cfm?id_ area $=830$ (acessado em 13/Jan/2009).

17. Bosch AM. Classical galactosaemia revisited. J Inherit Metab Dis 2006; 29:516-25.

18. Holton J, Walter J, Tyfield L. Galactosemia. In: Scriver CR, Beaudet AL, Sly WS, Valle D, Childs B, Kinzler KW, et al., editors. The metabolic and molecular bases of inherited disease. $8^{\text {th }}$ Ed. New York: McGraw-Hill; 2001. p. 1553-87.

19. Elsas LJ. Galactosemia. In: Pagon RA, Bird TC, Dolan CR, Stephens K, editors. GeneReviews. http://www.ncbi.nlm.nih.gov/books/NBK1518/ (acessado em 14/Jan/2011).

20. Applegarth DA, Toone JR, Lowry RB. Incidence of inborn errors of metabolism in British Columbia, 1969 - 1996. Pediatrics 2000; 105:e10.

21. Padilla CD, Dans LF, Estrada SC, Tamondong Jr. MR, Laceste JJ, Bernal RM. Cost-benefit analysis of newborn screening for galactosemia in the Philippines. Southeast Asian J Trop Med Public Health 2003; 34 Suppl 3:215-20.

22. Henderson H, Leisegang F, Brown R, Eley B. The clinical and molecular spectrum of galactosemia in patients from the Cape Town region of South Africa. BMC Pediatr 2002; 2:7.

23. Departamento de Informática do SUS. Informações de saúde. Estatísticas vitais. http://www2. datasus.gov.br/DATASUS/index.php?area $=0205$ (acessado em 10/Jan/2011).

24. Camelo Jr. JS, Fernandes MIM, Maciel LMZ, Scrideli CA, Santos JLF, Camargo Jr. AS, et al. Galactosaemia in a Brazilian population: high incidence and cost-benefit analysis. J Inherit Metab Dis 2009; Epub ahead of print.

25. Brosnan CA, Swint JM. Cost analysis: concepts and application. Public Health Nurs 2001; 18:13-8.

26. Bachmann C, Colombo JP. Incidence of disorders tested by systematic screening: confidence limits and comparison of programmes. J Inherit Metab Dis 1982; 5:3-5.

27. Banco Central do Brasil. PEDD: Padrão Especial de Disseminação de Dados. http://www.bcb.gov.br/ pec/sdds/port/sddsp.htm?perfil=1 (acessado em 20/Mai/2009).

28. Davis JP, Eisenhardt KM, Bingham CB. Developing theory through simulation methods. Acad Manage Rev 2007; 32:480-99.

29. Vanni T, Luz PM, Ribeiro RA, Novaes HMD, Polanczyk CA. Avaliação econômica em saúde: aplicações em doenças infecciosas. Cad Saúde Pública 2009; 25:2543-52.
30. Banco Central do Brasil. Copom: 143a reunião. http://www.bcb.gov.br/?COPOM143 (acessado em 30/Jun/2009).

31. Regional Office for the Western Pacific, World Health Organization. Assessing new vaccines for national immunization programmes: a framework to assist decision makers. Manila: World Health Organization; 2000.

32. World Health Organization. Community genetic services in Latin America and regional networks on medical genetics. Report of a WHO Collaborating Centre. Geneva: World Health Organization; 2004.

33. Canada JR, Sullivan WG, White JA. Capital investment analysis for engineering and management. Upper Sadle River: Prentice Hall; 1996.

34. Dinwiddy CL, Teal FJ. Principles of cost-benefit analysis for developing countries. New York: Cambridge University Press; 1996.

35. Schulpis K, Papakonstantinou ED, Michelakakis H, Podskarbi T, Patsouras A, Shin Y. Screening for galactosaemia in Greece. Paediatr Perinat Epidemiol 1997; 11:436-40.

36. Fundação Sistema Estadual de Análise de Dados. População por sexo, raça/cor, Estado de São Paulo, 2004. http://www.seade.gov.br/produtos/ idr/dem/dem_pop_03.htm (acessado em 05/ Set/2008).

37. Margutti AVB. Análise vetorial de impedância bioelétrica e ângulo de fase de recém-nascidos a termo, adequados para a idade gestacional [Dissertação de Mestrado]. Ribeirão Preto: Faculdade de Medicina de Ribeirão Preto, Universidade de São Paulo; 2009.

38. Magalhães PKR, Turcato MF, Ângulo IL, Maciel LMZ. Programa de Triagem Neonatal do Hospital das Clínicas da Faculdade de Medicina de Ribeirão Preto, Universidade de São Paulo, Brasil. Cad Saúde Pública 2009; 25:445-54.

39. Luz GS, Carvalho MD, Pelloso SM, Higarashi IH. Prevalence of diseases diagnosed by the Program of Neonatal Screening in Maringa, Paraná, Brazil: 2001-2006. Rev Gaúch Enferm 2008; 29:446-53.

40. Waisbren SE, Read CY, Ampola M, Brewster TG, Demmer L, Greenstein R, et al. Newborn screening compared to clinical identification of biochemical genetic disorders. J Inherit Metab Dis 2002; 25:599600 .

41. Walter JH. Arguments for early screening: a clinician's perspective. Eur J Pediatr 2003; 162 Suppl $1: S 2-4$.

42. Clague A, Thomas A. Neonatal biochemical screening for disease. Clin Chim Acta 2002; 315:99-110.

43. Carroll AE, Downs SM. Comprehensive cost-utility analysis of newborn screening strategies. Pediatrics 2006; 117(5 Pt 2):S287-95.

44. Carvalho TM, Santos HP, Santos IC, Vargas PR, Pedrosa J. Newborn screening: a national public health programme in Brazil. J Inherit Metab Dis 2007; 30:615.

45. Borrajo GJ. Newborn screening in Latin America at the beginning of the 21st century. J Inherit Metab Dis 2007; 30:466-81.

46. Alfonso I, Charria G. Updating neonatal neurometabolic screening. Medicina (B.Aires) 2009; 69:36-40. 
47. Loeber JG. Neonatal screening in Europe; the situation in 2004. J Inherit Metab Dis 2007; 30:430-8.

48. Williams M. Neonatal screening for metabolic diseases: need for efficacy studies. Ned Tijdschr Geneeskd 2008; 152:1653-6.

49. Therrell BL, Adams J. Newborn screening in North America. J Inherit Metab Dis 2007; 30:447-65.

50. National Newborn Screening and Genetics Resource Center. National newborn screening status report. http://genes-r-us.uthscsa.edu/nbsdisor ders.pdf (acessado em 10/Set/2009).
51. Canadian Organization for Rare Diseases/National Newborn Screening and Genetics Resource Center. National newborn screening status report. http://genes-r-us.uthscsa.edu/CA_nbsdisorders. pdf (acessado em 15/Set/2009).

52. Padilla CD, Therrell BL. Newborn screening in the Asia Pacific region. J Inherit Metab Dis 2007; 30:490-506.

Recebido em 01/Jul/2009

Versão final reapresentada em 10/Fev/2011 Aprovado em 22/Fev/2011 